\title{
Status of the COROT ground-based photometric activities
}

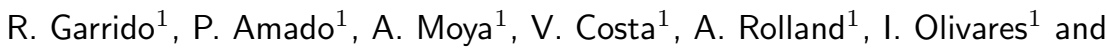 \\ M. J. Goupil ${ }^{2}$
}

1.-Instituto de Astrofisica de Andalucia, Apdo. 3004, Granada, Spain

2.-DASGAL, UMR CNRS 8633, Observatoire de Paris-Meudon, France

\begin{abstract}
We report photometric observations made at the Observatorio de Sierra Nevada of the secondary targets in the input catalogue of COROT. These observations consist of Strömgren -uvby- and Crawford $-H \beta$ - measurements of stars in Scenarios 1, 2 and 4. The cooler stars are being measured in the CaII $H \& K$ photometric system. We present here the present status of observations and reductions together with some preliminary results on the monitoring of photometric variability for some selected stars.
\end{abstract}

\section{Introduction}

In order to have an homogeneous data base of the secondary targets (up to $8 \mathrm{mag}$ ) it was decided to perform photometric and spectroscopic observations of the preliminary COROT selected fields. Here we present the photometric observations carried out in the four band-uvby- Strömgren system, where as usual:

$(b-y)$ is basically a temperature indicator

$m=(v-b)-(b-y)$ is a metallicity indicator

and

$c=(u-v)-(v-b)$ is a luminosity indicator

and in the Crawford $-H \beta$ - system where

$H \beta$ is another independent temperature indicator for most of the star sample, hence the reddening will be calculated using the $H \beta$ index.

The use of the CaII $H \& K$ band has been introduced since it is more than two times more sensitive to metallicity changes than $m_{1}$, with sensitivity 


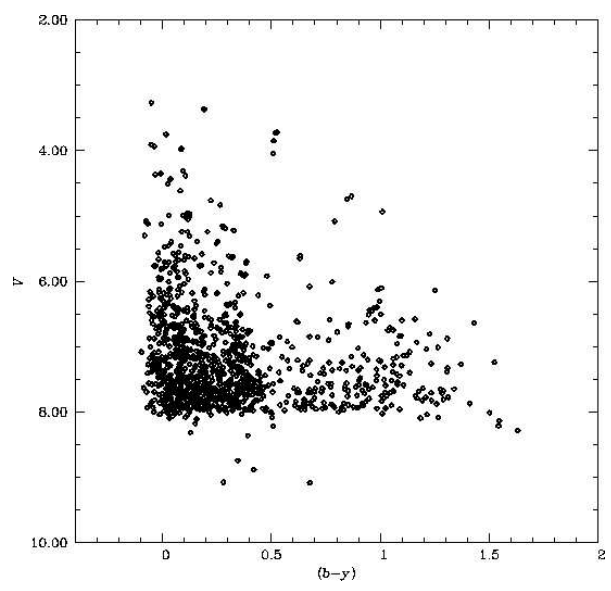

Figure 1: $V$ versus $(b-y)$ color diagram. $(b-y)$ is unreddened. Stars fainter than $8 \mathrm{mag}$. are probably missidentifications of multiple systems.

increasing to lower temperatures, as shown by Twarog and Anthony-Twarog (1991).

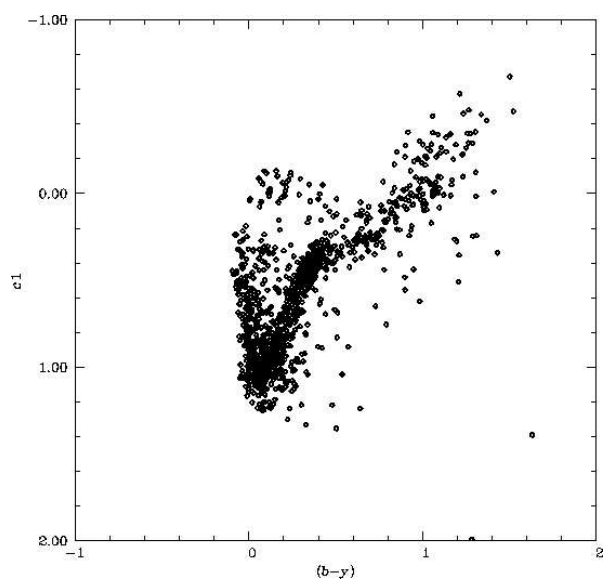

Figure 2: $c$ versus $(b-y)$ color diagram. Colors are unreddened. 


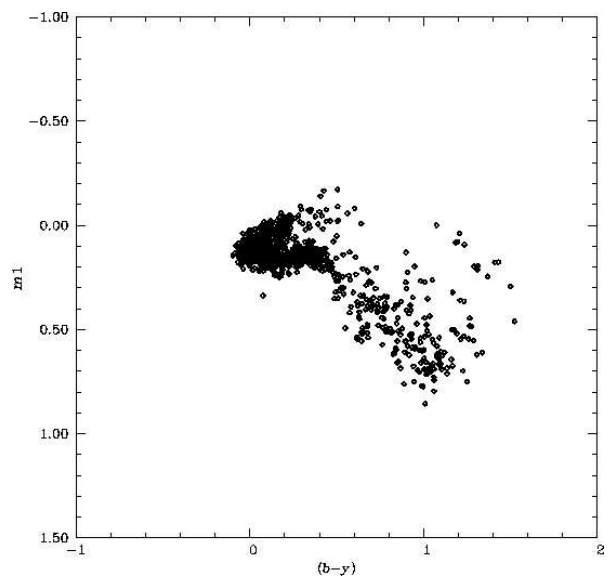

Figure 3: $m$ versus $(b-y)$ color diagram. Colors are unreddened.

\section{Instrumentation}

All the Strömgren and Crawford observations were made with a photoelectric photometer able to work in two modes: in the Strömgren mode, it measures simultaneously the four uvby bands and in the Crawford mode, it measures simultaneously two bands nw (narrow and wide) centered on the $H \beta$ line. This photometer is attached to a $0.9 \mathrm{~m}$ telescope on Sierra Nevada Observatory, property of the IAA (CSIC) situated near the city of Granada. The building contains two domes hosting two telescopes. CaII H\&K observations were made with a CCD camera attached to the other telescope with a diameter of $1.5 \mathrm{~m}$. Details of this instrumentation can be downloaded from http://www.iaa.es/osn/principal/html.

\section{Catalogue observations}

The observations we present here began in early 2000 when it was not yet known the final scenario chosen for COROT, at that time there was two possibilities: one starting at $\alpha=5 \mathrm{~h} 37 \mathrm{~m}$ (and 12h later) called scenario 1 and other starting at $\alpha=7 \mathrm{~h} 19 \mathrm{~m}$ (and $12 \mathrm{~h}$ later) called scenario 2 . We decided to observe both scenarios in order to have more physical information on the possible targets to help in the final selection. After some discussions during the meeting of the Scientific Committee held in Paris in spring 2001 an intermediate scenario was finally decided for COROT: scenario 4, starting at $\alpha=6 \mathrm{~h} 03 \mathrm{~m}$ and ending at $7 \mathrm{~h} 31 \mathrm{~m}$ (and $12 \mathrm{~h}$ later), always within an equatorial band of \pm 10 degrees. This 


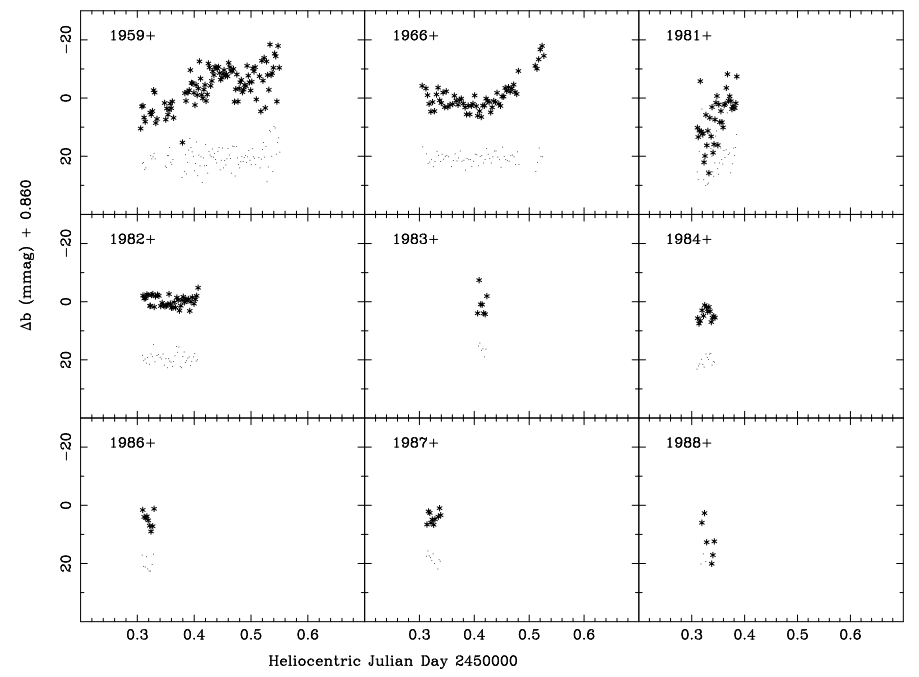

Figure 4: Photometric variability of HD 49434. Stars are magnitude differences between HD 49434 and HD 48922. Points are for HD 49933 minus HD 48922.

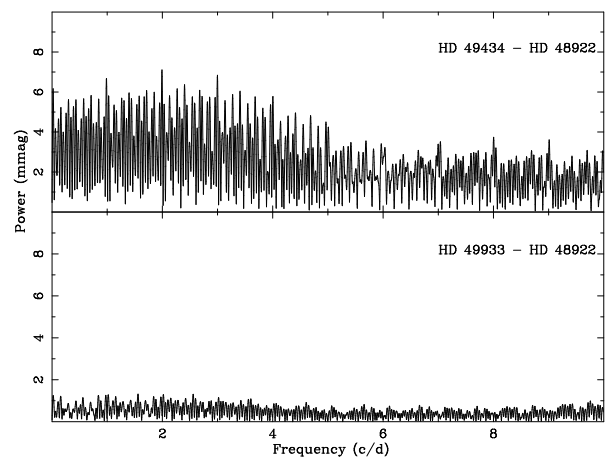

Figure 5: Power spectra of both photometric series shown in Figure 4.

final scenario has some stars in common with scenarios 1 and 2 but most of the stars were new.

Before the decision was taken to select scenario 4 most of the stars in scenarios 1 and 2 were observed. In particular for scenario 1, 539 stars out of 661 were observed in the Strömgren and Crawford systems and 78 out of 144 in the $C a I I H \& K$ system. For scenario 2 these numbers were: 442 out of 458 and 69 out of 128 . We started the new observations for scenario 4 in summer 2001 and all the visible stars in the season were observed in Strömgren and 
Table 1: Fitted frequencies of HR 6534.

$\begin{array}{rrrrr}\nu(\mu \mathrm{Hz}) & \text { Amp (mag) } & \text { phase }(\mathrm{rad}) & \nu_{i} / \nu_{0} & \nu_{i} / \nu_{1} \\ 158.92 & 0.0022 \pm 0.0003 & 3.40 \pm 0.16 & 1 & - \\ 207.45 & 0.0039 \pm 0.0004 & 4.57 \pm 0.09 & 0.77 & 1 \\ 237.38 & 0.0018 \pm 0.0004 & 1.47 \pm 0.19 & 0.67 & 0.87 \\ 291.95 & 0.0022 \pm 0.0004 & 4.84 \pm 0.16 & 0.56 & 0.71 \\ 353.76 & 0.0029 \pm 0.0003 & 0.25 \pm 0.12 & 0.45 & 0.59\end{array}$

Crawford systems: a total of 155 objects. No observations were made in the CaII $H \& K$ system during this summer.

In conclusion, 155 out of 419 stars have been observed in scenario 4 in the Stömgren and Crawford systems and 105 out of 187 stars were observed in the $C a I I H \& K$ system. We expect to finish all the observations in summer 2002 , including the remaining stars from scenarios 1 and 2 in order to have an homogeneous catalogue to compare with spectroscopic observations made also for stars of these two scenarios.

We present in Figure 1, 2 and 3 some preliminary and partial results of the photometric reductions now in progress.

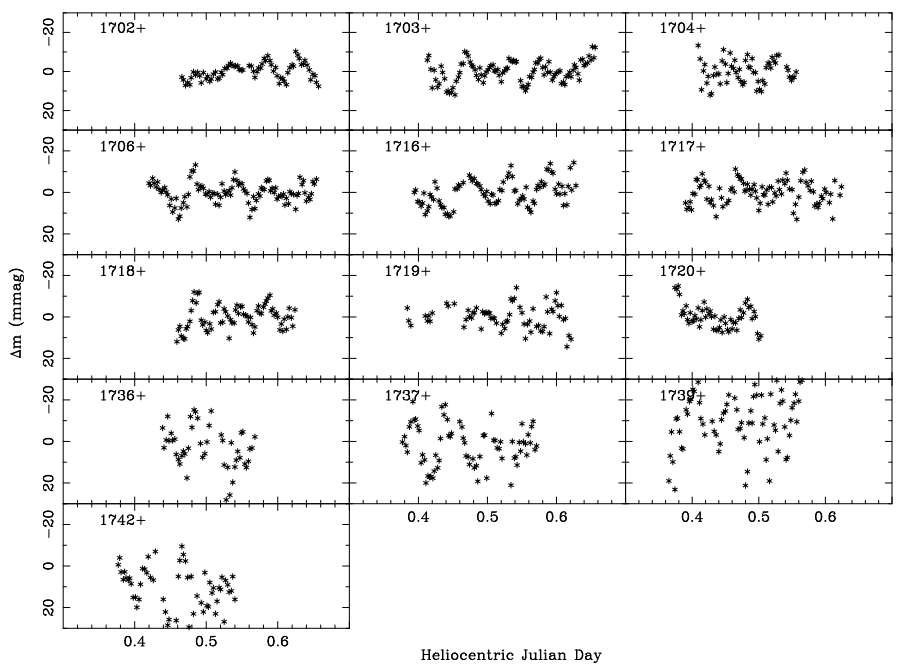

Figure 6: $(b+v)$ photometric variations of HR 6534. 
Table 2: Frequency of the fundamental radial mode and period ratios for radial modes of a $1.75 M_{\odot}$ model with no rotation $(v=0)$ and with a rotational velocity of $v=100 \mathrm{~km} / \mathrm{s}$ and $v=255 \mathrm{~km} / \mathrm{s}$.

$\begin{array}{rrrrrrrr}v(\mathrm{~km} / \mathrm{s}) & \log T_{\text {eff }} & \nu_{0}(\mu \mathrm{Hz}) & P_{1} / P_{0} & P_{2} / P_{0} & P_{3} / P_{0} & P_{4} / P_{0} & P_{5} / P_{0} \\ 0 & 3.90 & 207.2 & 0.772 & 0.6305 & 0.533 & 0.458 & 0.399 \\ 100^{b} & 3.90 & 210.6 & 0.777 & 0.633 & 0.536 & 0.462 & 0.398 \\ 0 & 3.89 & 188.2 & 0.772 & 0.628 & 0.530 & 0.456 & 0.398 \\ 100^{b} & 3.89 & 191.2 & 0.782 & 0.627 & 0.534 & 0.460 & 0.397 \\ 250^{a} & 3.89 & 203.4 & 0.787 & 0.643 & 0.545 & 0.473 & 0.416 \\ 250^{b} & 3.89 & 221.2 & 0.884 & 0.736 & 0.635 & 0.553 & 0.488\end{array}$

a no degenerate coupling included

tb degenerate coupling included

\section{Photometric monitoring}

Some bright stars from scenarios 1,2 or 4 were observed to be slightly variable in spectroscopy by Claude Catala and then selected to be monitored in photometry. From these HD 171834, HD 164259, HD 46304, HD 174866 and HD 180868 were found to be "non-variable" during one night of observation ( 6 hours aproximately) at the level of 2 mmags rms (see Figure 5b for a typical power spectrum of a "non-variable" star). Only HD 49434 showed some photometric variability as can be seen in Figure 4. This long term variability, see power spectrum in Figure 5, is typical for the newly discovered $\gamma$ Dor stars as discussed in Bruntt et al. (2002).

One of the comparison stars to test variability turned out to be a new $\delta$ Scuti star: HD $159170=$ HR 6534. Light curves are plotted in Figure 6. The star is clearly multiperiodic and a preliminary Fourier solution is shown in Table 1.

Period ratios seem to indicate that HR 6534 is a pure radial pulsator but the star has a rotational velocity of $255 \mathrm{kms}^{-1}$ and when high rotation is included in the pulsation model the radial overtones do not show anymore the classical period ratios as shown in Table 2. More observations have been made during 2001 from OSN and from SAT in La Silla in order to confirm these results that will be discussed in a forthcoming paper by Garrido et al. 2002.

Unfortunateley HR 6534 (or "Orianita" as MJG wants it to be renamed) is outside the scenario 4 ( $\alpha=17 \mathrm{~h} 33 \mathrm{~m} 30$ s and $\delta=-05 \mathrm{~d} 44 \mathrm{~m} 41 \mathrm{~s})$

\section{References}

Anthony-Twarog, B. J., Twarog, B. A. 1995, AJ 109, 2828

Bruntt, H., Catala, C., Garrido, R., Rodriguez, E. 2002, in preparation

Garrido, R., Goupil, M. J., et al. 2002, in preparation 